\title{
THE
}

\section{Transcranial focal electrical stimulation via tripolar concentric ring electrodes does not modify the short- and long-term memory formation in rats evaluated in the novel object recognition test}

\author{
G. Rogel-Salazar \\ University of Rhode Island \\ H. Luna-Munguía \\ University of Rhode Island \\ K. E. Stevens \\ Walter G. Besio \\ University of Rhode Island, besio@uri.edu \\ Follow this and additional works at: https://digitalcommons.uri.edu/ele_facpubs \\ This is a pre-publication author manuscript of the final, published article. \\ Creative Commons License
}

(c) (i) $\odot$

This work is licensed under a Creative Commons Attribution-Noncommercial-No Derivative Works 4.0 License.

\begin{abstract}
Citation/Publisher Attribution
Rogel-Salazar, G., Luna-Munguía, H., Stevens, K. E., \& Besio, W. G. (2014). Transcranial focal electrical stimulation via tripolar concentric ring electrodes does not modify the short- and long-term memory formation in rats evaluated in the novel object recognition test. Epilepsy \& Behavior, 27(1), 154-158. doi: 10.1016/j.yebeh.2013.01.006

Available at: https://doi.org/10.1016/j.yebeh.2013.01.006
\end{abstract}

This Article is brought to you for free and open access by the Department of Electrical, Computer, and Biomedical Engineering at DigitalCommons@URI. It has been accepted for inclusion in Department of Electrical, Computer, and Biomedical Engineering Faculty Publications by an authorized administrator of DigitalCommons@URI. For more information, please contact digitalcommons-group@uri.edu. 


\title{
Transcranial focal electrical stimulation via tripolar concentric ring electrodes does not modify the short- and long-term memory formation in rats evaluated in the novel object recognition test
}

\author{
G Rogel-Salazar ${ }^{1}$, H Luna-Munguía ${ }^{1}$, KE Stevens ${ }^{2,3}$, and WG Besio ${ }^{1,{ }^{*}}$ \\ ${ }^{1}$ Electrical, Computer, and Biomedical Engineering, University of Rhode Island, 4 East Alumni \\ Ave., Kingston, Rhode Island 02881, USA \\ ${ }^{2}$ Dept. of Psychiatry, University of Colorado, Denver, Anschutz Medical Campus, Aurora, \\ Colorado 80045, USA \\ ${ }^{3}$ Medical Research Service, Veterans Affairs Medical Center, Denver, Colorado, 80220;USA
}

\begin{abstract}
Noninvasive transcranial focal electrical stimulation (TFS) via tripolar concentric ring electrodes (TCREs) has been under development by Besio as an alternative/complementary therapy for seizure control. TFS has shown efficacy attenuating penicillin, pilocarpine, and pentylenetetrazole- induced acute seizures in rat models. This study evaluated the effects of TFS via TCREs on the memory formation of healthy rats as a safety test of TFS. The short and longterm memory formation was tested after the application of TFS using the novel object recognition (NOR) test. Independent groups were used: naïve, control (without TFS), and TFS (treated). Naïve, control, and stimulated groups spent more time investigating the new object than the familiar one during the test phase. TFS via TCREs given once does not modify the short- and long-term memory formation in rats in the NOR test. Results provide an important step towards a better understanding for the safe usage of TFS via TCREs.
\end{abstract}

\section{Keywords}

Transcranial focal electrical stimulation; TFS; Tripolar concentric ring electrodes; TCRE; shortterm memory; long-term memory; novel object recognition test; NOR

\section{Introduction}

Brain stimulation is a promising new technology for the treatment of medically intractable epilepsy. However, most brain stimulation techniques involve invasive procedures to implant electrodes and electronic stimulators [for a review on various brain stimulation techniques for epilepsy see [1]. In contrast, noninvasive electrical stimulation does not

\footnotetext{
(C) 2012 Elsevier Inc. All rights reserved

"Corresponding author to: Electrical, Computer, and Biomedical Engineering, University of Rhode Island, 4 East Alumni Ave., Kingston, Rhode Island 02881, USA. Tel. (401) 874-4738. besio@ele.uri.edu.

Publisher's Disclaimer: This is a PDF file of an unedited manuscript that has been accepted for publication. As a service to our customers we are providing this early version of the manuscript. The manuscript will undergo copyediting, typesetting, and review of the resulting proof before it is published in its final citable form. Please note that during the production process errors may be discovered which could affect the content, and all legal disclaimers that apply to the journal pertain.
} 
require the risks of implantation, and the electrodes can be moved easily as needed to determine where they may be the most effective in reducing seizure activity [2].

Besio has been developing noninvasive transcranial focal electrical stimulation (TFS) via tripolar concentric ring electrodes (TCREs) as an alternative/complementary therapy for seizure control. This innovative noninvasive stimulation technique has demonstrated excellent efficacy with penicillin, pilocarpine and pentylenetetrazole - induced rat seizure models [2-4]. Furthermore, when the scalp of the rat was analyzed results showed that TFS via TCREs did not damage it [5] or the underlying cortex [6].

The short and long-term side effects of TFS are not completely understood. It is possible to study the safety of the electrical stimulation in the brain through the analysis of their functional consequences on the memory formation $[7,8]$. We are interested in demonstrating that TFS via TCREs has no undesirable effects on the memory formation and is safe per se. The aim of this study was to evaluate the effects of the TFS via TCREs on the memory process of healthy rats. To explore this issue, we addressed the following question: what are the functional consequences of applying noninvasive TFS via TCREs on the shortand long-term memory formation, tested in the novel object recognition (NOR) test in healthy rats.

The NOR test has become the task of choice for assessing aspects of declarative memory in rodents [9-11]. It has been widely demonstrated that spontaneous exploratory activity in the rat can be used to provide a valid measure of memory function [10]. The NOR test exploits the natural tendency of rats to explore novel stimuli in preference to familiar stimuli [10, 12], and gives information on working, short-term or long-term memory depending on the elapsed testing phase [13]. For example, during the test phase, the memory formation could be tested for short-term (the first $90 \mathrm{~min}$ ) and long-term (24-48h) memory [9]. Advantages of the NOR test includes no pre-training and involves no explicit reinforcement (such as food or electric shocks) $[9,10,12]$.

\section{Material and methods}

\subsection{Subjects}

Male Sprague-Dawley rats (weighing 250-300 g) were ordered from Harlan Laboratories (Madison, WI) and housed in groups of $2-3$ subjects in polycarbonate cages $(48.2 \times 26.6 \times$ 20.3) with bedding material (7092 Corncob, Harlan Laboratories Inc., Madison, WI). They were kept under $12: 12 \mathrm{~h}$ cycle conditions and a room temperature of $24 \pm{ }^{\circ} \mathrm{C}$. All behavioral tests were conducted between 1000 and $1400 \mathrm{~h}$. Subjects were provided with free access to water and rat chow (2020SX Teklad Global 18\% soy protein-free extruded rodent diet sterilizable, Harlan Laboratories Inc., Madison, WI) throughout the experiments. At the end of the study, rats were euthanized by $\mathrm{CO}_{2}$ inhalation. The experimental protocol was approved by the University of Rhode Island IACUC.

\subsection{Novel Object Recognition (NOR) Test}

2.2.1. Apparatus-The NOR test was performed in a blue acrylic opaque open field chamber $(60 \times 60 \times 60 \mathrm{~cm})($ Clever System Inc. $)$ with faint black-painted squares $(15 \times 15$ $\mathrm{cm})$. The open field chamber was placed on a table $(80 \mathrm{~cm}$ from the floor $)$ in a dark room illuminated only by a $60 \mathrm{~W}$ light bulb mounted $1 \mathrm{~m}$ above the area. White-noise source from one extraction hood provided constant background noise $(72 \mathrm{~dB})$. A video camera mounted directly above the box was used to record the testing session. Behaviour was videotaped for later manual scoring. 
2.2.2. Objects-The familiar objects, and duplicates, were made of glass. The familiar object was a clean copy of the two identical objects used during the familiarization phase, thus ensuring that the familiar object had not been scent-marked during the familiarization phase. The novel objects varied in shape and color and were made of plastic. Preliminary observations showed that rats had no exploration preference between objects (plastic vs. glass). All the rats were tested with the same objects. The sizes of the objects were no smaller than the size of the rat and no larger than 2.5 times the size of the rat [12]. The objects were secured to the floor of the open field chamber using Velcro strips, which also served as marks that ensured that the objects were always placed in the same location within the open field [14].

2.2.3. Habituation-During the habituation phase, each rat was handled (rats were gently held by the experimenter from tail and body) for 5 min each day for 5 consecutive days. After 30 min of handling, rats were placed inside the acrylic opaque open field chamber (always facing to the opposite wall where objects were placed later) and allowed to explore and become familiar with the empty arena (context) for $5 \mathrm{~min}$. No object was placed inside the box during habituation. The open field chamber was carefully cleaned with $60 \%$ alcohol prior to habituation of the next rat.

2.2.4. Testing-Testing consisted of four phases presented in the following order: (1) Rehabituation, (2) Familiarization, (3) Delay and (4) Test. The behavior was videotaped for later scoring. Between each phase the box and objects were cleaned with $60 \%$ alcohol to avoid odour trails.

1. Re-habituation: Each rat was placed in the empty open field and allowed to explore for $1 \mathrm{~min}$. Afterwards, animals were removed from the box and placed in their home cage (for $1 \mathrm{~min}$ ) meanwhile two equal objects were put in the arena.

2. Familiarization: One minute later after re-habituation, rats were returned to the open field and allowed to explore two identical objects for $3 \mathrm{~min}$.

3. Delay: During the delay rats were removed from the open field chamber (and placed into their home cage) and the familiar object was paired with a novel object. Delay times were: $10 \mathrm{~s}, 1 \mathrm{~min}, 10 \mathrm{~min}, 90 \mathrm{~min}, 24 \mathrm{~h}$ and $48 \mathrm{~h}$.

4. Test: After completion of the delay interval, the rats were placed back in the open field chamber and allowed to explore the two objects for $3 \mathrm{~min}$. Exploration was defined as the animal directing the nose within $2 \mathrm{~cm}$ of the object while looking at or sniffing the object. Exploration was not scored when the rat climbed on top of the object or if another part of the rat's body touched the object. The recognition index (RI) was used to evaluate cognitive function. RI was calculated by dividing the novel object exploration time by the total exploration time (novel/novel + familiar investigation) [15]. Values of RI close to 0.5 indicate that animals spent equal time exploring both objects (familiar and the novel), while RI values greater than 0.5 denote a preference to explore the novel object over the familiar one.

\subsection{Application of noninvasive TFS via TCRES}

On the day prior to the NOR test the rat scalp was shaved. The day of the experiment, subjects were held by one researcher while another used conductive paste to apply the TCREs on the scalp. Rats were randomly assigned to the control and treatment groups. Only the treatment group received TFS via TCREs. The TFS was applied immediately after the familiarization phase. 
The parameters and methods for the TFS via TCREs used in this experiment were based on our previous studies that have shown efficacy attenuating penicillin, pilocarpine, and pentylenetetrazole- induced acute seizures in rat models [2-4]. One TCRE was placed at the top center of the head. Flexible cables connected the TCREs to the stimulator. The TFS via TCRE was given once according to these specifications: $2 \mathrm{~min}, 300 \mathrm{~Hz}, 200 \mu \mathrm{s}$ equal biphasic pulses at $50 \mathrm{~mA}$. The control group was fully instrumented like the treatment group, but did not receive TFS.

\subsection{Stimulation System}

The stimulator was custom designed and built by our group with frequency, phase, and time duration of the TFS output signals programmable. The magnitude of the stimulation is adjusted manually. The stimulation controller, a Basic Stamp 2P (Parallax, Inc), was preprogrammed to apply TFS automatically when triggered. The TFS was programmed for charge balance to improve safety.

\subsection{Locomotor Activity Test}

The locomotor activity was evaluated during the evaluation of memory, the number of times the subject crossed with all paws from one square to another (crossings) was counted during 3 -min periods. The open field chamber was carefully cleaned between tests with $60 \%$ alcohol [16].

\subsection{Experimental groups}

For evaluating the effects of TFS on memory, three groups were needed: Naïve, Control (without TFS), and TFS (treated). The naïve group $(n=12)$ received habituation for handling, familiarization in the empty open field, and evaluation with the NOR test. Animals for control and TFS groups ( $\mathrm{n}=12$ and 13, respectively) received habituation for handling, familiarization with the empty open field, and also habituation for the TFS procedure. The control group received faked TFS, and only the TFS group was administered TFS immediately after the familiarization phase. The delay intervals were chosen to assess the specific memory types: short-term memory (10 sec, 1, 10 and 90 minutes) and long-term memory (24 and 48 hours) $[12,13]$.

\subsection{Statistical analyses}

The results are expressed as the mean \pm standard error of the mean (S.E.M.). A two-way repeated analysis of variance (ANOVA) followed by the Holm-Sidak test was performed to analyze differences between delays (or groups) and objects in the NOR test. Here groups are: Naïve vs. Control vs. TFS groups. The locomotor activity tested differences within the naïve, control, and treated groups and were analyzed using the one-way analysis of variance (ANOVA) followed by the Holm-Sidak test. A $P$ value of less than 0.05 was considered significant. Sigma Plot with Sigma Stat Integration (version 9.0, Systat Software, Inc., San Jose, California, USA) was used for all statistical analyses.

\section{Results}

\subsection{Familiarization Phase}

Figure 1 shows that during the familiarization phase, animals exhibited a comparable amount of time exploring the two identical objects in the naïve, control and TFS. There was no main effect of group $\left(\mathrm{F}_{(2,22)}=0.39, P=0.68\right)$ or object $\left(\mathrm{F}_{(1,22)}=0.61, P=0.45\right)$ nor was there a group $\mathrm{x}$ object interaction $\left(\mathrm{F}_{(2,22)}=1.02, P=0.37\right)$. 


\subsection{Test Phase}

Figure 2 shows the recognition index (RI) during the test phase for the naïve, control and stimulated groups in the object recognition test. The naïve, control, and treated groups showed more preference for exploring the novel object than the familiar one at all the delay times (10 s, $1 \mathrm{~min}, 10 \mathrm{~min}, 90 \mathrm{~min}, 24 \mathrm{~h}$ and 48h). The two-way repeated analysis of variance (ANOVA) did not find significant differences for the factor group $\left(\mathrm{F}_{(2,110)}=1.37\right.$, $P=0.275$ ) and the interaction between factors (Group-Time; $\mathrm{F}_{(10,110)}=1.49, P=0.152$ ) but showed differences for the factor time $\left(\mathrm{F}_{(5,110)}=3.01, P=0.018\right)$.

\subsection{Locomotor activity}

Table 1 shows the locomotor activity evaluation during the NOR test. During the familiarization phase naïve, control, and TFS groups had similar levels of locomotor activity $\left(\mathrm{F}_{(2,34)}=0.018, P=0.981\right)$. During the test phase in the delay of $10 \mathrm{~s}$ and $1 \mathrm{~min}$ all groups (naïve, control, and TFS) significantly decreased their locomotor activity relative to their familiarization phase (Holm-Sidak test $P<0.05$ ). The control and TFS groups, at $10 \mathrm{~s}$ delay $\left(\mathrm{F}_{(2,34)}=12.27, P<0.001\right)$ and the TFS group at 1 min delay $\left(\mathrm{F}_{(2,34)}=3.61, P=0.038\right)$ significantly reduced their locomotor activity in comparison to the naïve group. The locomotor activity in all groups (naïve, control and TFS) for the delay of $48 \mathrm{~h}$ significantly increased relative to their familiarization phase (Holm-Sidak test $P<0.05)$.

\section{Discussion}

In this study we found that the TFS via unique TCREs does not modify the short- and longterm memory formation in healthy rats evaluated with the NOR test. These results suggest that short- and long-term memory formation are not affected by the TFS via TCREs which provide a promising step towards a better understanding of its safe usage.

\subsection{Effect of applying noninvasive TFS via TCREs on the memory formation}

When a subject is familiar with an object, the subject will recognize the familiar object when exposed to it again; this is called recognition memory [13]. The recognition memory of naïve rats was assed in order to establish basal conditions for our experiment. Our results demonstrated that naïve rats showed more preference for novel objects than familiar objects. This observation is in agreement with the literature; the NOR paradigm is based on the natural tendency of rodents to explore new objects more -preference of novelty- in comparison to familiar objects [9-11]. These results verify that naïve animals displayed good memory performance under our experimental conditions.

The control group (similar to the naive group) showed higher exploration towards the novel object than the familiar object. The control group received placebo TFS via TCREs. This result suggests that the habituation to the procedure of TFS via TCREs does not affect the memory performance of the animals.

The main goal of this experiment was to establish the functional consequences of applying noninvasive TFS via TCREs on the memory formation. The present data showed that the TFS via TCREs does not modify the short- and long-term memory formation in healthy rats evaluated with the NOR test. This idea is supported by the fact that animals that received TFS via TCREs spent more time exploring the novel object than the familiar one (as also was exhibited in the naïve and control groups). These results constitute the first report that TFS via TCREs do not produce adverse effects in the memory formation. 


\subsection{Brain stimulation and memory formation}

It is difficult to make comparisons of the effects of our TFS via TCREs on the memory formation to invasive electrical stimulation or even with other techniques of noninvasive brain stimulation. In general, some reports mention that invasive and noninvasive electrical stimulation induce augmentation of the memory formation while others indicate no apparent undesirable effects [17-25].

The deep brain stimulation (DBS, invasive technique) has been demonstrated to improve or at least not show apparent undesirable effects on the memory formation. For example, using the autoshaping task, the high frequency electrical (HFS) stimulation applied in the hippocampus produced an augmentation in the short-term but not in the long-term memory formation in healthy rats [17]. Also, the effects of DBS applied in the hippocampus of patients with temporal lobe epilepsy have shown no modifications in the short-term memory formation [18].

Similar to the invasive brain stimulation, transcranial magnetic stimulation (TMS) and transcranial direct current stimulation (tDCS) (both noninvasive techniques) have been shown to enhance the memory process while not exhibiting adverse memory modifications $[7,19,20]$. For example, in healthy rats, the evaluation of the visuospatial working memory after applying tDCS in the frontal cortex demonstrated that the stimulation had no effect on the short-term memory but showed a long-term benefit (animals exhibited significantly more efficient place avoidance and skill retention in comparison to the controls) [21]. In healthy humans after applying anodal tDCS in the prefrontal cortex, results demonstrated that this stimulation enhanced the working memory performance while cathodal tDCS interfered with it [22].

The use of repetitive transcranial magnetic stimulation (rTMS) has been shown to improve the animal's performance in the NOR test at high frequency $(15 \mathrm{~Hz})$ and to impair the memory formation at lower frequency $(1 \mathrm{and} 8 \mathrm{~Hz})$ in healthy mice [23]. Also studies evaluating the effect of TMS on the cognitive functions in humans are still controversial; results are not sufficiently conclusive to assert that the TMS enhances the memory process $[24,25]$.

When comparing stimulation techniques, several factors should be considered to evaluate the effects that invasive/noninvasive brain stimulation has on the memory formation: a) structure stimulated [hippocampus, prefrontal cortex, thalamus, etc]; b) characteristics of the electrical stimulation; c) evaluation of short- or long-term memory formation; d) which tests are used for evaluating the memory process; e) studies in humans or animal models; f) healthy or pathological subjects, etc.

\subsection{Effect of applying noninvasive TFS via TCREs on the locomotor activity}

One procedure that helps to evaluate the levels of anxiety-like behaviors in rodents is through the quantification of the locomotor activity in the open field chamber [16]. The NOR test gives the opportunity to evaluate the memory formation and at the same time the locomotor activity. Taking advantage of this possibility, we assessed the locomotor activity of the animals. Decrease/increase of the total locomotion activity is interpreted as an anxiolytic/anxiogenic-like effect, respectively [16].

All groups of animals that were submitted to the NOR test exhibited an increase in their anxiety levels during the first minute. One explanation for observing this anxiogenic-like effect is that the first minute of exposing the animals to a novel environment with objects is a very high stressful situation. In contrast, all groups displayed an anxiolytic-like effect in the $48 \mathrm{~h}$ delay. This result could reflect that the animal's levels of anxiety-like behavior 
diminished due to the repetition of submitting them to the open field chamber. Despite the modification in the locomotor activity all the subjects showed an increased exploration of the novel object over the familiar one.

\subsection{Final considerations}

It is important to be critical about the precision with which TFS via TCREs can target specific parts of the brain. Presently, we can not assert that the electrical field was focally concentrated in a specific part of the rats' brain or if the rats received a generalized electrical stimulation. One preliminary report of our group indicates that the extra-cranial TFS current would be sufficient to cause the activation of neurons in the hippocampus [26]. Moreover future experiments should be carried out to determine what structures are being stimulated.

One limitation of this study is that prior to testing memory, the TFS via TCREs was applied on the scalp for two minutes only once. Previously, we proposed TFS via TCREs as a novel alternative/complementary therapy for seizure control where the TFS was triggered once or twice to stop PTZ-induced electrographic activity [27, 28]. In clinical practice, the application of the TFS via TCREs may need to be given more than once per day. More experiments are necessary to evaluate the consequence of repetitive application of TFS via TCREs in the memory formation under normal and pathological conditions.

In conclusion, TFS via TCREs given once does not modify the short and long-term memory formation in healthy rats tested in the NOR test. Considering that one dose of TFS on rat scalp [5] and multiple applications on cortex [6] caused no significant damage, along with these current findings on eloquent brain formation in behaving rats, the application of TFS seems to be safe. However, further research should be executed to understand the effect of applying TFS via TCREs on memory formation.

\section{Acknowledgments}

The project described was supported in part by award number R21NS061335 from the National Institute of Neurological Disorders and Stroke and award number R21TW009384 by the Fogarty International Center of the National Institutes of Health. The content is solely the responsibility of the authors and does not necessarily represent the official views of the National Institutes of Health.

\section{References}

1. Theodore WH, Fisher RS. Brain stimulation for epilepsy. Lancet Neurol. 2004; 3:111-8. [PubMed: 14747003]

2. Besio WG, Gale KN, Medvedev AV. Possible therapeutic effects of transcutaneous electrical stimulation via concentric ring electrodes. Epilepsia. 2010; 51 (Suppl 3):85-7. [PubMed: 20618408]

3. Besio, WG.; Nayak, A.; Koka, K.; Jiang, W.; Sahin, M.; Patwardhan, R. Localized transcutaneous electrical brain stimulation development. Annual International Conference of the BMES; Baltimore, MD, USA. 2005. p. 1113

4. Besio WG, Koka K, Cole AJ. Effects of noninvasive transcutaneous electrical stimulation via concentric ring electrodes on pilocarpine-induced status epilepticus in rats. Epilepsia. 2007; 48:2273-9. [PubMed: 17651415]

5. Besio WG, Sharma V, Spaulding J. The effects of concentric ring electrode electrical stimulation on rat skin. Ann Biomed Eng. 2010; 38:1111-8. [PubMed: 20087776]

6. Mucio-Ramirez, S.; Makeyev, O.; Liu, X.; Leon-Olea, M.; Besio, W. Cortical integrity after transcutaneous focal electrical stimulation via concentric ring electrodes. Society for Neuroscience Annual Meeting; Washington, DC, USA. 2011. p. Abs. 672.20/Y19

7. Floel A, Cohen LG. Contribution of noninvasive cortical stimulation to the study of memory functions. Brain Res Rev. 2007; 53:250-9. [PubMed: 17023050] 
8. Mukamel R, Fried I. Human intracranial recordings and cognitive neuroscience. Annu Rev Psychol. 2012; 63:511-37. [PubMed: 21943170]

9. Clark RE, Squire LR. An animal model of recognition memory and medial temporal lobe amnesia: history and current issues. Neuropsychologia. 2010; 48:2234-44. [PubMed: 20144894]

10. Ennaceur A, Delacour J. A new one-trial test for neurobiological studies of memory in rats. 1: Behavioral data. Behav Brain Res. 1988; 31:47-59. [PubMed: 3228475]

11. Winters BD, Saksida LM, Bussey TJ. Object recognition memory: neurobiological mechanisms of encoding, consolidation and retrieval. Neurosci Biobehav Rev. 2008; 32:1055-70. [PubMed: 18499253]

12. Clark RE, Zola SM, Squire LR. Impaired recognition memory in rats after damage to the hippocampus. J Neurosci. 2000; 20:8853-60. [PubMed: 11102494]

13. Carlini, VP. The Object Recognition Task: A New Proposal for the Memory Performance Study, Object Recognition. Cao, Tam Phuong, editor. In Tech; 2011. p. 27-42.Available from: http:// www.intechopen.com/books/object-recognition/the-object-recognition-task-a-new-proposal-forthe-memory-performance-study

14. Broadbent NJ, Gaskin S, Squire LR, Clark RE. Object recognition memory and the rodent hippocampus. Learn Mem. 2009; 17:5-11. [PubMed: 20028732]

15. de Lima MN, Laranja DC, Bromberg E, Roesler R, Schröder N. Pre- or post-training administration of the NMDA receptor blocker MK-801 impairs object recognition memory in rats. Behav Brain Res. 2005; 156:139-43. [PubMed: 15474658]

16. Prut L, Belzung C. The open field as a paradigm to measure the effects of drugs on anxiety-like behaviors: a review. Eur J Pharmacol. 2003; 463:3-33. [PubMed: 12600700]

17. Luna-Munguía H, Meneses A, Peña-Ortega F, Gaona A, Rocha L. Effects of hippocampal highfrequency electrical stimulation in memory formation and their association with amino acid tissue content and release in normal rats. Hippocampus. 2012; 22:98-105. [PubMed: 20882549]

18. Velasco AL, Velasco M, Velasco F, Menes D, Gordon F, Rocha L, Briones M, Márquez I. Subacute and chronic electrical stimulation of the hippocampus on intractable temporal lobe seizures: preliminary report. Arch Med Res. 2000; 31:316-28. [PubMed: 11036183]

19. Reis J, Robertson EM, Krakauer JW, Rothwell J, Marshall L, Gerloff C, Wassermann EM, Pascual-Leone A, Hummel F, Celnik PA, Classen J, Floel A, Ziemann U, Paulus W, Siebner HR, Born J, Cohen LG. Consensus: Can transcranial direct current stimulation and transcranial magnetic stimulation enhance motor learning and memory formation? Brain Stimul. 2008; 1:3639.

20. Rossi S, Rossini PM. TMS in cognitive plasticity and the potential for rehabilitation. Trends Cogn Sci. 2004; 8:273-9. [PubMed: 15165553]

21. Dockery CA, Liebetanz D, Birbaumer N, Malinowska M, Wesierska MJ. Cumulative benefits of frontal transcranial direct current stimulation on visuospatial working memory training and skill learning in rats. Neurobiol Learn Mem. 2011; 96:452-60. [PubMed: 21763775]

22. Zaehle T, Sandmann P, Thorne JD, Jäncke L, Herrmann CS. Transcranial direct current stimulation of the prefrontal cortex modulates working memory performance: combined behavioural and electrophysiological evidence. BMC Neurosci. 2011; 12:1-12. [PubMed: 21208416]

23. Ahmed Z, Wieraszko A. Modulation of learning and hippocampal, neuronal plasticity by repetitive transcranial magnetic stimulation (rTMS). Bioelectromagnetics. 2006; 27:288-94. [PubMed: 16511879]

24. Robertson EM, Theoret H, Pascual-Leone A. Studies in cognition: the problems solved and created by transcranial magnetic stimulation. J Cogn Neurosci. 2003; 15:948-60. [PubMed: 14614806]

25. Sparing R, Mottaghy FM. Noninvasive brain stimulation with transcranial magnetic or direct current stimulation (TMS/tDCS)-From insights into human memory to therapy of its dysfunction. Methods. 2008; 44:329-37. [PubMed: 18374276]

26. Besio WG, Hadidi R, Makeyev O, Luna-Munguía H, Rocha L. Electric fields in hippocampus due to transcranial focal electrical stimulation via concentric ring electrodes. Conf Proc IEEE Eng Med Biol Soc. 2011:5488-91. [PubMed: 22255580]

27. Besio, W.; Koka, K.; Gale, K.; Medvedev, A. Preliminary data on anticonvulsant efficacy of transcutaneous electrical stimulation via novel concentric ring electrodes. Advances in the 
application of technology to epilepsy: the CIMIT/NIO. Epilepsy Innovation. In: Schachter, SC.; Guttag, J.; Schiff, SJ.; Schomer, DL., editors. Epilepsy Behav. Vol. 16. 2009. p. 3-46.

28. Makeyev O, Liu X, Luna-Munguía H, Rogel-Salazar G, Mucio-Ramirez S, Liu Y, Sun YL, Kay SM, Besio WG. Toward a noninvasive automatic seizure control system in rats with transcranial focal stimulations via tripolar concentric ring electrodes. IEEE Trans Neural Syst Rehabil Eng. 2012; 20:422-31. [PubMed: 22772373] 


\section{Highlights}

- The effect of focal stimulation was evaluated with a novel object recognition test

- Noninvasive focal electrical stimulation does not modify memory formation

- Noninvasive electrical stimulation via concentric ring electrodes is safe on rats 


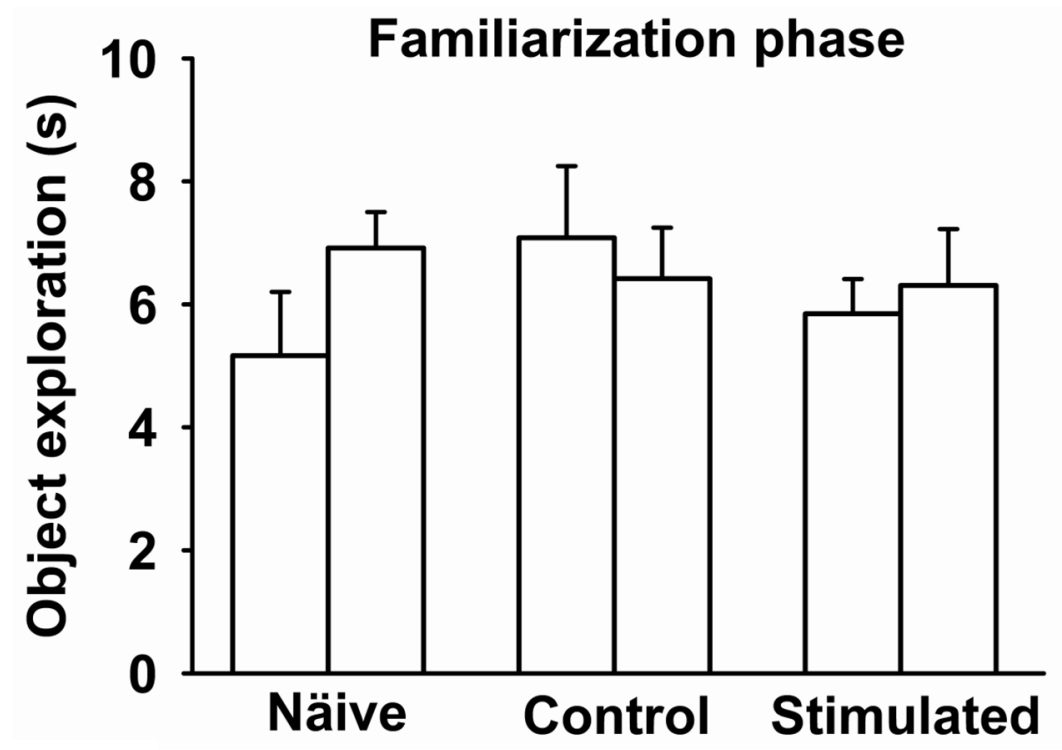

Fig. 1.

During the familiarization phase all groups of rats (naïve, control and stimulated) showed a comparable amount of time exploring two equal objects evaluated in the novel object recognition test. Data are presented as the mean \pm the S.E.M. $(n=12-13)$. 


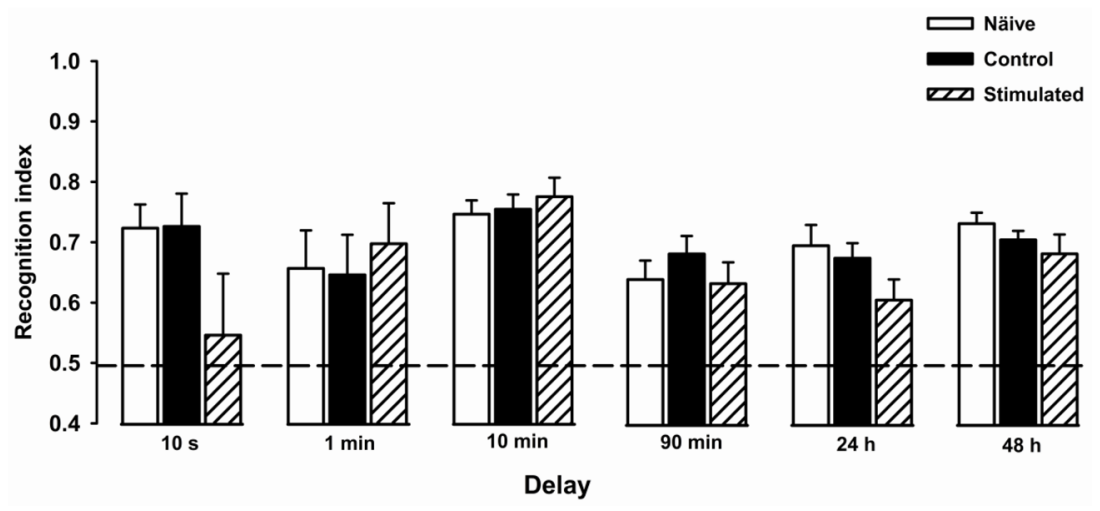

Fig. 2.

Effect of transcranial focal stimulation via tripolar concentric ring electrodes on memory performance (expressed as recognition index) of rats tested in the novel object recognition test. Animals were stimulated immediately after the familiarization phase and tested later according to the delay intervals for evaluating short-term memory (10 sec, 1, 10 and 90 minutes) and long-term memory ( 24 and 48 hours). Data are presented as the mean \pm the S.E.M. (n=12-13). 
